\title{
3D RECONSTRUCTION OF CORONARY ARTERIES FROM ROTATIONAL X-RAY ANGIOGRAPHY
}

\author{
Chaima Oueslati, Sabra Mabrouk, Faouzi Ghorbel \\ National School of Computer Science \\ CRISTAL laboratory \\ GRIFT research group \\ Manouba 2010 \\ Tunisia
}

\author{
Mohamed Hedi Bedoui \\ Faculty of Medecine of Monastir \\ TIM Team \\ Laboratory of Biophysics \\ Monastir 5019 \\ Tunisia
}

\begin{abstract}
X-ray angiography has been an effective modality for diagnosing coronary artery disease representing one of the leading causes of death. A 3D reconstruction of the coronary arteries is a very important step to facilitate the interpretation of angiograms. In this paper, we propose a 3D reconstruction method of the coronary arteries. In order to improve the pairwise matching performance of our approach, we introduce artificial interest points (nodes) to respect the topology variation between $3 \mathrm{D}$ vascular trees. New similarity measures are proposed to take into consideration the non-rigid coronary artery movement. To measure the performance of the proposed method, we evaluate the proposed method on clinical data. Results show that accuracy of vessel centerlines has the average projection error equal to $0.5 \mathrm{~mm}$ for 28 different patients.
\end{abstract}

\section{Keywords}

X-Ray angiography, 3D reconstruction, 3D vascular trees, artificial nodes

\section{INTRODUCTION}

Coronary heart disease has been for decades one of the primary dangers to human health essentially due to coronary atherosclerosis. In clinical practice, several image acquisition techniques were developed for cardiac examination, the most currently used one is the $\mathrm{X}$-ray angiography thanks to the temporal and spatial resolutions of the provided images and the speed of the medical examination associated with it. However, the 2D nature of produced images makes it difficult to interpret the overlaying and crossing structures.

The sequence of projections thus available can be exploited to perform a $3 \mathrm{D}$ reconstruction of the coronary tree that, associated with $3 \mathrm{D}$ visualization tools and quantification, will be able to bring a substantial help in documentation of injuries and search for optimal incidences of observation in which the interventional cardiologist can perform the angioplasty procedure. This $3 \mathrm{D}$ reconstruction, however, represents a real challenge because of structures movement.

Permission to make digital or hard copies of all or part of this work for personal or classroom use is granted without fee provided that copies are not made or distributed for profit or commercial advantage and that copies bear this notice and the full citation on the first page. To copy otherwise, or republish, to post on servers or to redistribute to lists, requires prior specific permission and/or a fee.
Among the rich literature on coronary artery reconstruction [1], two main categories have been proposed model-based methods (modelling) and tomographic reconstruction.

The first inspired by the principles of computer vision exploits a modeling technique by proceeding the mapping of extracted 2D entities from 2 to 4 projections [2], This method try to find a binary representation of the 3D/4D structure of the coronary arteries. In order to perform the reconstruction, authors in [7] compute the $2 \mathrm{D}$ vessel centerlines from the projections and then search correspondences in them to represent the motion without using the ECG signal.

Authors in [8] use an optimisation scheme from two uncalibrated angiographic images with 14 parameters included by minimizing two errors distances and direction vectors of corresponding skeleton points in projections, then they use the epipolar constraints and the vessel surface is extracted fitting elliptical cross-sections to carry out the correspondence of centerline points between the two projections.

While the second categorie proceeds by static tomographic reconstruction from a weak number of projections. In fact, tomographic reconstruction methods aim at reconstructing 3D / 4D volume of attenuation coefficients [3].

The forward projections are computed using the $3 \mathrm{D}$ reconstructions of markers on the guidewire in [9] and the ECG-gated tomographic reconstruction in [10]. In some cases, the forward projected images are prepared 
to extract some features (e.g. centrelines) for the registration [10].

With the movement related to the breathing of the patient and the heart, the branches undergo additional nonlinear deformations and they are different for each patient. Two similar branches from one phase to another do not have the same curvature or the same coordinates. This problem makes it difficult to map arteries for each phase. These variations can be due to various difficulties related to acquired 2D images (presence of noise, reduction of contrast medium, structures superposition, etc.), the movement of the heart, the breathing of the patient or to differences related to the angle of view. In [5], authors describe an inaccurate tree matching algorithm for recording $3 \mathrm{D}$ trees of nonisomorphous coronary arteries over time.

In this paper, a 3D reconstruction method of coronary arteries from two projections of monoplanes, uncalibrated and acquired under two different projection angles is described. Based on geometric characteristics and the characteristics of the nodes, the similarity between nodes is calculated using new criteria. A step which takes into account the variation of topology between 2D vascular trees is presented and propose to insert artificial interest points. In our case, the skeleton matches are not found point by point using the epipolar constraints, but branch to branch using the similarity measure between the extremity nodes of two branch in the $2 \mathrm{D}$ sequence.

The rest of this paper is organized as follows. In section 2 , the proposed method is described in detail. In section 3 , experimental results are presented and conclusions are draw in the last section.

\section{PROPOSED METHOD}

From the 2D skeletons of the segmented image, the 3D structure of the vascular tree can be extracted. In our case, the reconstruction is done on a pair of projections. Based on the information of the central line, a 2D tree is deduced for each segmented image [4]. Then, each remarkable point such as the bifurcation point and the end point is considered as a point of interest. From one projection to another, a branch can actually disappear, appear or be of different length depending on the heart phase.

The framework of the proposed method is shown in Fig.1.

\subsection{Matching approach}

\subsubsection{The similarity measure}

We proposed two metrics to determine the similarity of the interest points in the two projections. The new node-to-node metrics allow taking into account nonrigid movements of the coronary arteries.

We note that instead of choosing a system of absolute coordinates linked to the acquisition room, we prefer a relative presentation of the one projection according to the other, as a simplification, the first image is considered as a reference, the passage of one view to another is reacted by a rigid transformation composed of a $3 *$ 3 rotation matrix $\mathrm{R}$ and a $3 * 1$ translation matrix $\mathrm{T}$.

Similarity measures significantly influence pairwise matching results. The choice of the node-to-node metrics is important, moreover, between two coronary trees representing no completely successive phases of the cardiac cycle, the movement could be important therefore these metrics need to be as efficient as possible.

The Euclidean distance between the coordinates of two nodes in different trees can be used since the coronary trees are extracted from the same acquisition of the same patient.

The first metric is the Euclidean distance between a node after applying the rigid transformation in the first image and a node in the second image. In fact, we want to determine the closest node to the second node after applying the rigid transformation.

To compute the second metric, we first test if the two nodes have the same type (the two nodes are bifurcation points or endpoints), and if they have the same number of neighbors of type bifurcation and endpoint. If the two points verify these two conditions, two distances are calculated, the first is the sum of the Euclidean distances between the bifurcation neighbors and the second is the sum of the Euclidean distances between the ends neighbors.

The similarity measure represents the minimum pondered sum of the first metric and the second metric. We note that based on the choice of constraints and their measure threshold value, we obtain the best matching result which is only composed of a pair of tree similar nodes.

\subsubsection{Insertion of artificial nodes}

In the proposed method, we consider that the matching accuracy is more important than the corresponding node number. In order to refine the matching process by adding artificial nodes to take into account the topology variation between the vascular trees, we apply the Artificial nodes insertion algorithm [6].

These artificial nodes correspond to bifurcation points and leaf nodes that only exist in one of the vascular trees.

Figure 2 shows two examples of artificial nodes insertion. We treat the artificial node insertion in two cases: 


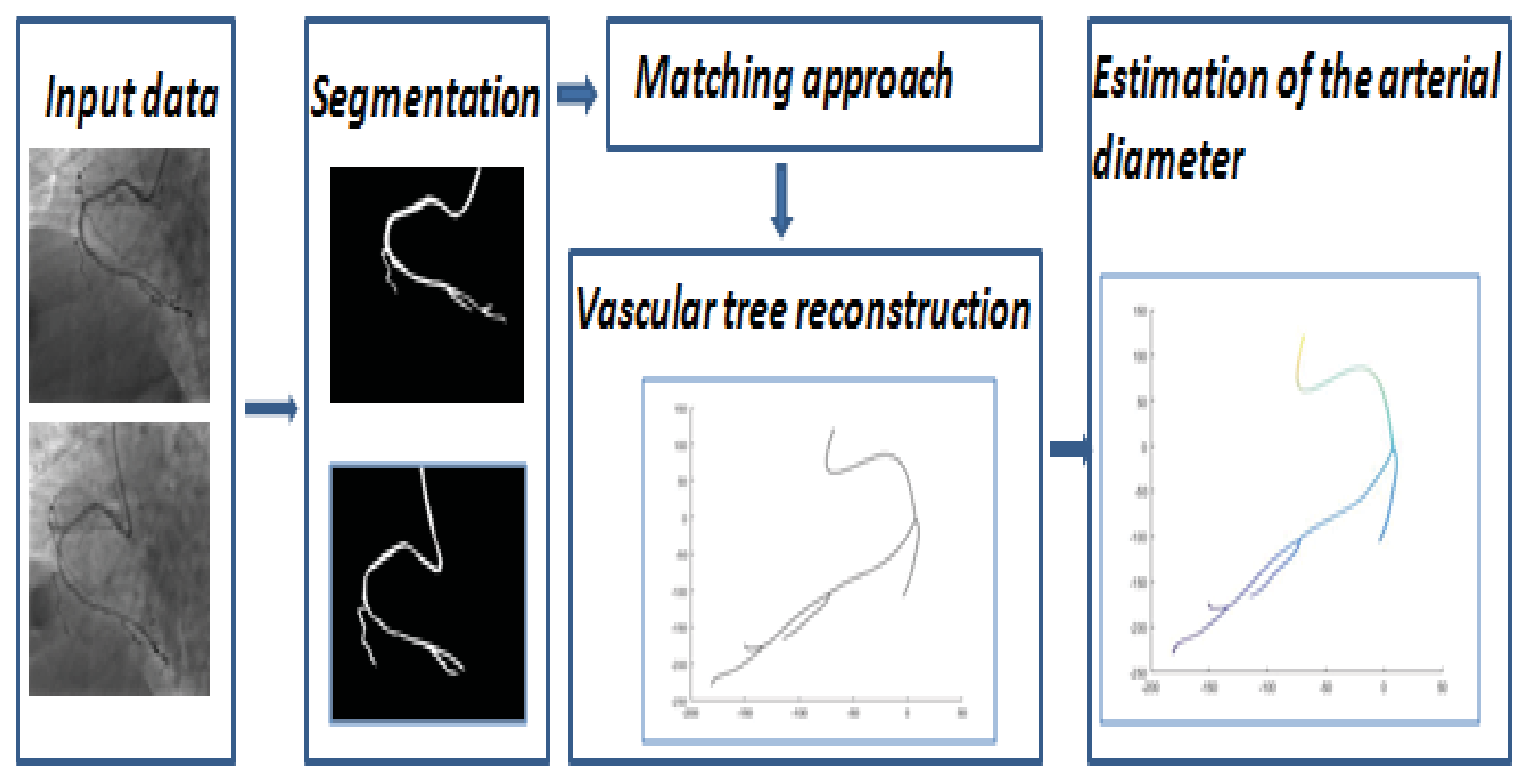

Figure 1: The framework of the proposed method

the artificial end nodes insertion in the first row and artificial bifurcation node insertion in the second row.

\subsection{Vascular tree Reconstruction}

After exhaustive merging, we are left with a set of threedimensional points that reflects the underlying anatomy, every two connected three-dimensional points represent a $3 \mathrm{D}$ branch. As we mentioned in the previous section, two similar branches from one phase to another do not have the same curvature, the same coordinates, and the same length.

In order to respect the topology of the arterial tree, an additional step is added making correspondence between particular points of each similar branches.

\subsubsection{Matching two branches' points}

We intend to make the branch-length reparameterization and extract the same number of points from each branch. For each point $p_{i}$, its correspondent point $p_{j}$ is the closest one in the other branch after applying the rigid transformation.

Given the matched nodes between centerline points in the two projections, three-dimensional reconstruction is deduced straight-forward using triangulation [12].

Once the 3D centerline established, we estimate the artery diameter with the method proposed in [4].

\section{EXPERIMENTAL RESULT}

We evaluate the proposed method on clinical data provided by Mongi-Slim Hospital of La Marsa Tunisia.
The datasets consist of 28 patients having each 8 to 12 images. The metric used for the evaluation of the proposed method is the average of the distances between the initial and reconstructed skeletons. This metric can be seen as the difference value between back projection results and original image pixels. it represents the sum of distance error between all back projection nodes $X^{P}$ of image and the original nodes $X$ :

$$
e=\sum_{i=1}^{N}\left(X_{i}^{P}-X_{i}\right)^{T}\left(X_{i}^{P}-X_{i}\right)
$$

Where $e$ is the re-projection error and $N$ is the total number of nodes.

The optimization procedure is performed by the Levenberg-Marquardt algorithm [11]. The idea is to decompose the objective function in the form of quadratic substitution functions, which makes the minimization simple and "parallelizable" from a computer point of view.// The average error of projection was reduced from $2.8 \mathrm{~mm} \pm 1.55$ before the optimization to $0.5 \mathrm{~mm} \pm 0.011$ after(Fig 3).

We note that the threshold associated with the similarity measure is empirical chosen and identical for each correspondence test. To determine the best configuration, we check if all the nodes pairs that need to be matched are selected.

The program execution is optimized thanks to the recursion of the mapping method which makes the execution time very fast. 


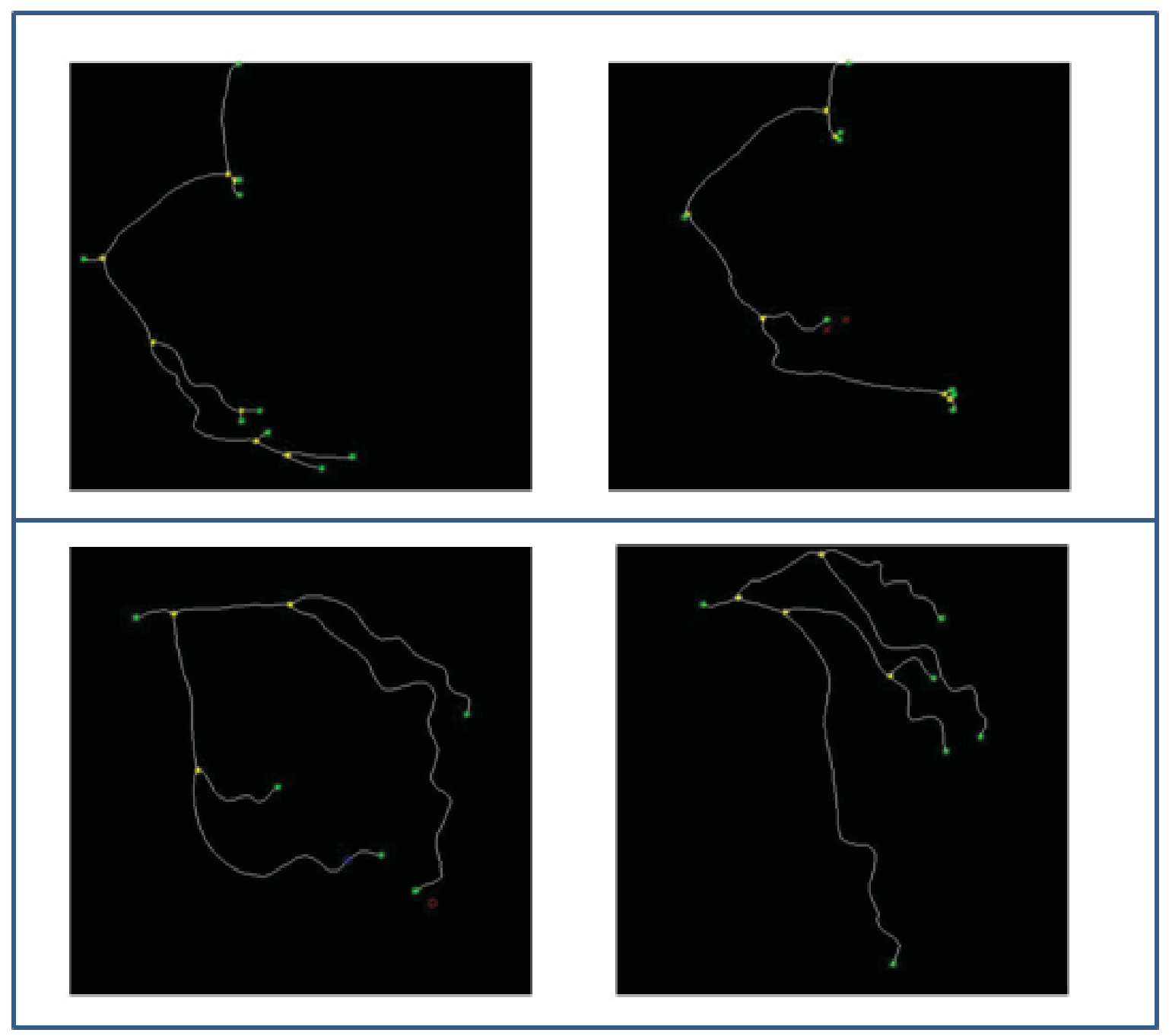

Figure 2: Insertion of artificial nodes: First row: insertion of artificial end points (red cross), second row: insertion of bifurcation node (blue circle) and end point (red circle)

\section{CONCLUSION}

In this paper, we proposed a $3 \mathrm{D}$ reconstruction method of the coronary tree from two projections of monoplanes, uncalibrated and acquired under two different projection angles. The geometric characteristics and the characteristics of the nodes are used to evaluate the similarity between the nodes and the edges using two new criteria. To take into account the variation of topology between coronary trees due to their extraction, a step which consists of adding an artificial node to our coronary trees is introduced.

\section{REFERENCES}

[1] ÇIMEN, Serkan, GOOYA, Ali, GRASS, Michael, et al. Reconstruction of coronary arteries from $\mathrm{X}$-ray angiography: A review. Medical image analysis, 2016, vol. 32, p. 46-68.
[2] CHEN, S.-YJ et CARROLL, John D. Kinematic and deformation analysis of 4-D coronary arterial trees reconstructed from cine angiograms. IEEE transactions on medical imaging, 2003, vol. 22, no 6, p. 710-721.

[3] SCHOONENBERG, Gert, FLORENT, Raoul, LELONG, Pierre, et al. Projection-based motion compensation and reconstruction of coronary segments and cardiac implantable devices using rotational X-ray angiography. Medical image analysis, 2009, vol. 13, no 5, p. 785-792.

[4] MABROUK, S., OUESLATI, C., et GHORBEL, F. Multiscale Graph Cuts Based Method for Coronary Artery Segmentation in Angiograms. IRBM, 2017, vol. 38, no 3, p. 167-175.

[5] FEUILlÂTRE, H., NUNES, J.-C., et TOUMOULIN, C. An improved graph matching algorithm for the spatio-temporal matching of 


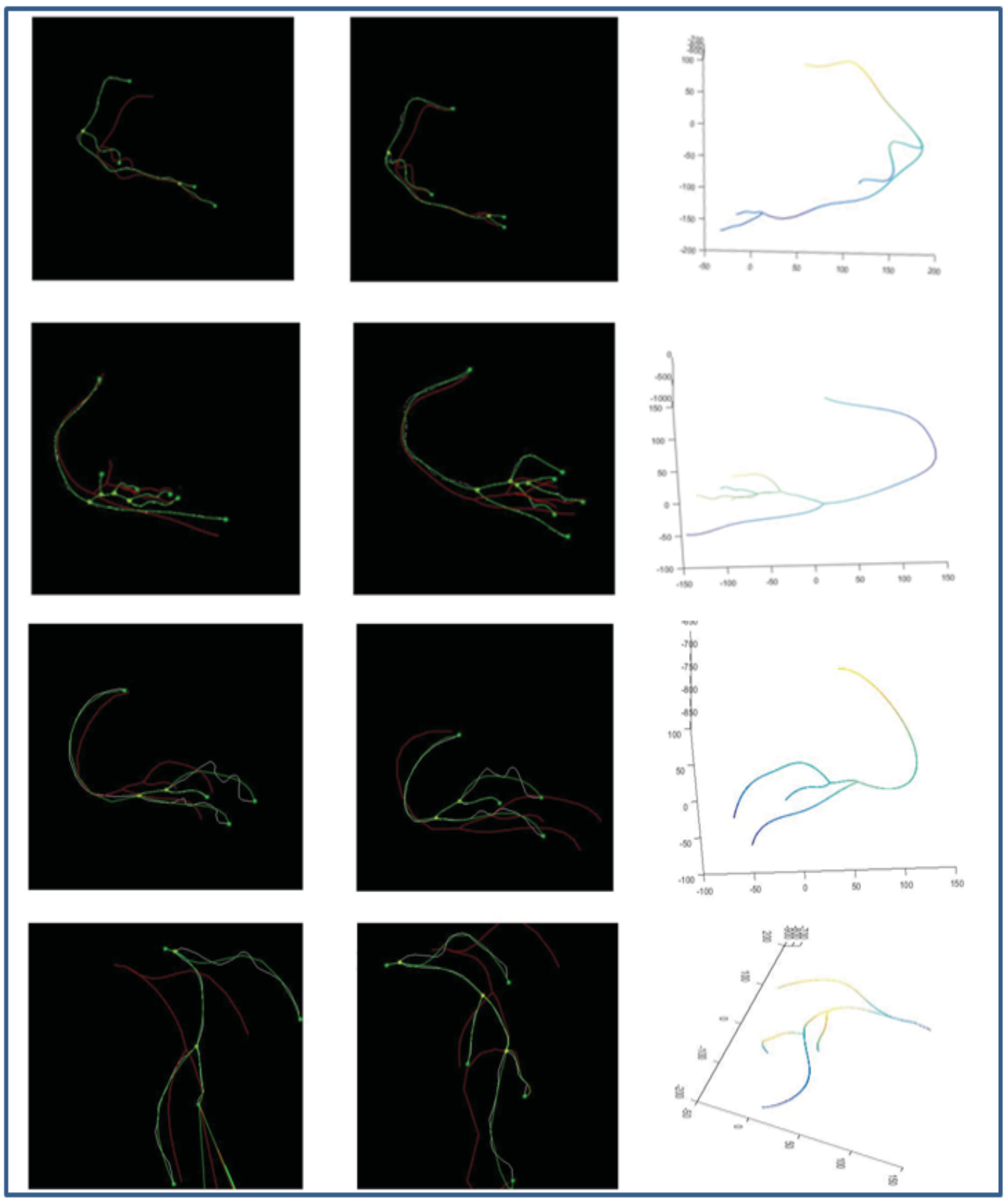

Figure 3: Reprojections of reconstruction results: (column 1 et 2): Erroneous, reprojected,and ground truth centerlines are overlapped in red, green and white respectively. (column 3) 3D reconstructed arteries tree 
a coronary artery 3D tree sequence. IRBM, 2015, vol. 36, no 6, p. 329-334.

[6] FEUILLÂTRE, Hélène, NUNES, Jean-Claude, et TOUMOULIN, Christine. Inexact coronary tree matching algorithm with artificial nodes. In : Engineering in Medicine and Biology Society (EMBC), 2016 IEEE 38th Annual International Conference of the. IEEE, 2016. p. 4153-4156.

[7] BLONDEL, Christophe, MALANDAIN, Grègoire, VAILLANT, RÃCgis, et al. Reconstruction of coronary arteries from a single rotational $\mathrm{X}$-ray projection sequence. IEEE Transactions on Medical Imaging, 2006, vol. 25, no 5, p. 653-663.

[8] YANG, Jian, WANG, Yongtian, LIU, Yue, et al. Novel approach for 3-D reconstruction of coronary arteries from two uncalibrated angiographic images. IEEE Transactions on Image Processing, 2009, vol. 18, no 7, p. 1563-1572.

[9] MOVASSAGHI, Babak, RASCHE, Volker, FLORENT, R., et al. 3D coronary reconstruction from calibrated motion-compensated 2D projections. In : International Congress Series. Elsevier, 2003. p. $1079-1084$.

[10] HANSIS, Eberhard, SCHÄFER, D., DÖSSEL, O., et al. Projection-based motion compensation for gated coronary artery reconstruction from rotational x-ray angiograms. Physics in medicine and biology, 2008, vol. 53, no 14, p. 3807.

[11] MORÉ, Jorge J. The Levenberg-Marquardt algorithm: implementation and theory. In : Numerical analysis. Springer, Berlin, Heidelberg, 1978. p. 105-116.

[12] Hartley R, Zisserman A. Multiple View Geometry in Computer Vision. Cambridge: Cambridge university press; 2003. 